\section{POLARISATION OF LIGHT * VIII.}

A QUARTZ plate cut parallel to the axis, when examined with convergent light, gives curves in the form of hyperbolas. These curves are wider in proportion to the thinness of the plate, but if the plate be thick enough to render the curves moderately fine, the colour becomes very faint. They may, however, be rendered distinct by using homogeneous light. The dark and light parts exchange positions when the analyser is turned through $90^{\circ}$. Two such plates with their axes at right angles to one another give coloured hyperbolas perfectly visible with the white light. Plates of Iceland spar exhibit similar phenomena, but the lines and curves are far more closely packed.

If the plate be cut in a direction inclined at $45^{\circ}$ (or at any angle differing considerably from $0^{\circ}$ or $90^{\circ}$ ) to the axis, the curves are approximately straight lines perpendicular to the principal section of the plate. Two such plates placed with their principal planes at right angles to one another give straight lines bisecting the angle between the principal planes. On this principle Savart constructed the polariscope which bears his name. It consists of two such plates and an analyser, and forms a very delicate test of the presence of polarisation. The lines are, of course, always in the direction described

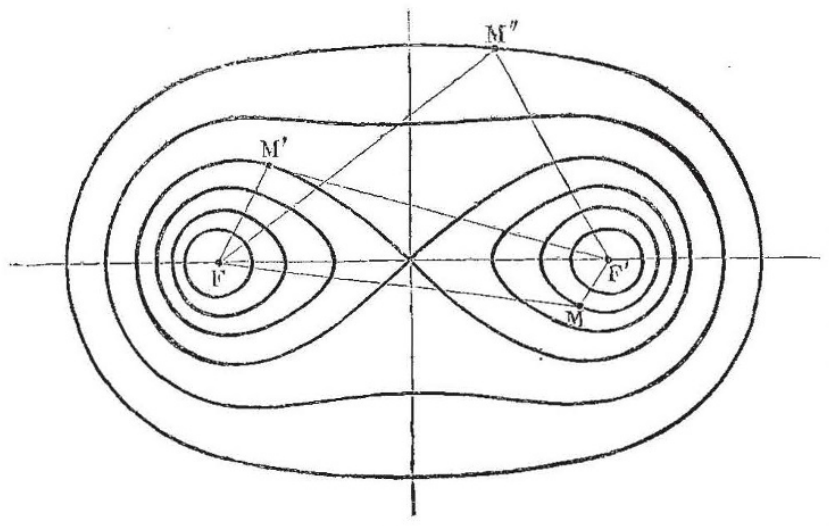

FIG. 26.

above, and the delicacy of the test increases in proportion as their direction becomes more and more nearly perpendicular to the original plane of vibration.

Bi-axal crystals exhibit a more complicated system of rings and crosses, or brushes as they may in this case be better termed. If such a crystal be cut in a direction perpendicular to the line which bisects the angle between the two optic axes (or the middle line, as it is called), the extremity of each of the axes will be surrounded with rings similar to those described in the case of the uni-axal crystals. The larger rings, however, are not strictly circles, but are distorted and drawn out towards one another; those which are larger still meet at a point midway between the centres, and form a figure of 8 , or lemniscata ; beyond this they form curves less and less compressed towards the crossing point, and approximate more and more nearly to an oval (see Fig. 26).

The vibrations of the two rays emerging from any point of a bi-axal crystal are as follows :- Of the two rays produced by the double refraction of a bi-axal crystal neither follows the ordinary law of refraction; but one does so more nearly than the other, and is on that account called for convenience the ordinary ray. And if through any point of the field of view we draw two lines to the points where the optic axes emerge, the directions

$$
\text { * Contirsed from p. } 466 \text {. }
$$

of vibration of the two rays will be those of the bisectors of the angles made by the two lines. If, therefore, the crystal be so placed that the line joining the extremities of the two axes coincides with the plane of vibration of either polariser or analyser, it is not difficult to see that there will be a black cross passing through the centre of the field, with one pair of arms in the line joining the extremities of the axes and the other pair at right angles to it. But if the plate be turned in its own plane round the central point, the points, for which the vibrations are parallel or perpendicular to those of the polariser or analyser, will no longer lie in straight lines passing through the centre, but will form two branches of a hyperbolic curve, passing respectively through the extremities of the optic axes.

If the analyser be turned round, the dark hyperbolic brushes, or the black cross, will undergo the changes analogous to those shown in the cross in the case of uniaxal crystals; but the most interesting effects are those seen when the polariser and analyser are crossed, and the crystal is turned in its own plane.

The angle between the optic axes in different kinds of crystals varies very much; in those where the angle is small it is easy to exhibit both at once in the field of view, but in others where the angle is large it is necessary to tilt the crystal so as to bring the two successively into view. In the latter case the crystal is sometimes cut in a direction perpendicular to one of the axes. The rings are then nearly circular, especially towards the centre, and in that respect they resemble those of a uni-axal crystal;

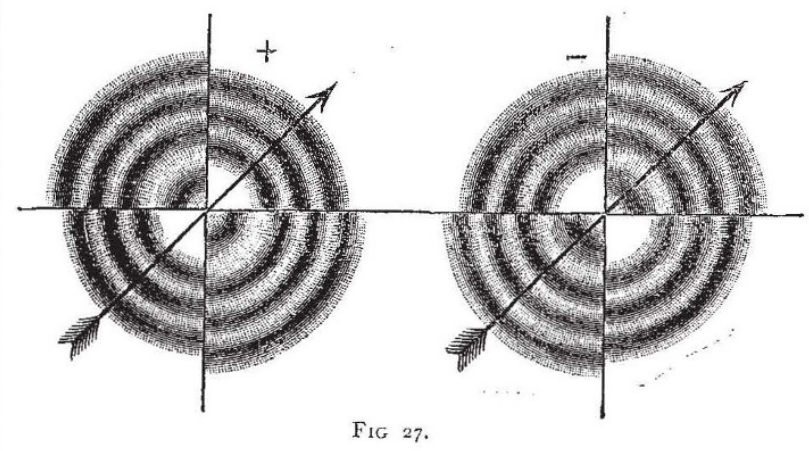

but the character of the specimen can never be mistaken because the rings are intersected by a black bar, or two arms in the same straight line, instead of by four arms at right angles to one another, as would have been the case if the crystal had been uni-axal. The following are the angles made by the optic waves in a few crystals :- -

\begin{tabular}{|c|c|c|}
\hline Carbonate & of le & \\
\hline Saltpetre & . & . \\
\hline Talc. & . & . \\
\hline Titanite & . & . \\
\hline Borax. & & \\
\hline Mica. & & . \\
\hline Carbonate & of & $\mathrm{im}$ \\
\hline Topaz of & razil & . \\
\hline Sugar & - & . \\
\hline Gypsum & . & . \\
\hline Felspar & & \\
\hline Topaz of & berd & \\
\hline Oxyde of I & ead & . \\
\hline Cyanite & . & - \\
\hline Chrysolite & . & . \\
\hline
\end{tabular}

These angles are determined by placing the crystal for an examination into an apparatus adapted to show the rings, and attaching it to an arm whereby the plate can be turned about an axis in its own plane. The axis is furnished with a circle divided into degrees and seconds, and an index. If this axis be horizontal, the plate is so 
placed that the line joining the centres of the two systems of rings is vertical, and the crystal is first turned so as to bring one centre into the centre of the field of view (usually marked by cross wires); the index is then read, and the crystal turned so as to bring the centre of the second system of rings to the centre of the field. The index is again read, and the difference of the two readings noted. This, however, gives not the true angle of the optic axes, but the apparent angle in air, that is, the angle between the rays as affected by refraction on emerging from the crystal. (See Fig. 27.)

In some crystals the optic axes have different angles of inclination for the different rays of the spectrum. Of this titanite or sphene is an example. All rays have a common middle line, and lie in the same plane, but the optic axes for the red rays are more widely separated than those for the blue, and consequently the part of the field which would exhibit a dark brush if red light were used is deprived of the red rays but not of the blue. The brushes, therefore, appear broader than with ordinary crystals, and are tinged with blue on the edges farthest from the middle point, and with red on the edges nearest to it. It is said that a similar distribution of the optic axes, or its opposite in which the red rays are least separated and the blue most, is found in all crystals belonging to the rhombic system.

In other crystals, the axes all lie in one plane, but all have not the same middle line, so that the two ring systems are unsymmetrical. This is the case with borax. In others the optic axes for different colours lie in different planes, all of which pass through the middle line.

Lastly, we may mention the crystals brookite and tartrate of ammonia soda and potash, in which the optic axes for the two extremities of the spectrum lie in planes at right angles to one another, both passing through the same middle line. If the systems of rings be examined with light which has been so widely dispersed that the portion illuminating the field in any given position is practically monochromatic, and the position of the instrument shifted through the different parts of the spectrum (or what is more convenient, if the different parts of the spectrum be successively thrown on the polariscope by means of a totally reflecting prism), the optic axes will be seen to draw gradually together until the figure closely resembles that of a uni-axal crystal; after which the axes open out in a direction at right angles to the former, until they have attained their greatest expansion. This experiment requires a strong light, butêtit is instructive, as show ing the exact distribution of the optic axes for different rays.

In some bi-axal crystals, rotably in gypsum, the distribution of the optic axes varies with the temperature. When the crystal is heated the angle between the optic axes diminishes until the crystal appears uni-axal; with a further increase of temperature the axes again open out, but in a direction at right angles to the former. When the crystal is cooled the axes generally resume their original directions. Sometimes, however, when the heating has been carried to a great degree, or has been continued for a long time, the axes never completely return to their normal condition; and in such a case the crystal may appear permanently uni-axal. Such an appearance, when permanent, has been considered a test of former heating; and this phenomenon, when presented by crystals found in a state of nature, may be taken as evidenco that the rocks in which they have been formed have been subject to high temperatures,

In the production and examination of the rings hitherto described, we have used light which has been plane-polarised and plane-analysed; but there is nothing to prevent our polarising the light or analysing it circularly, or indeed doing both.

If a quarter-undulation plate be placed between the polariser and the crystal to be examined, with its axis in- clined at $45^{\circ}$ to the plane of original vibration, the light will fall upon the plate in a state of circular polarisation; and as the polarisation will then have no reference to any particular plane of vibration, the black cross will disappear. A system of rings will be produced, but they will be discontinuous. At each quadrant, depending upon the position of the analyser, the rings will be broken, the portions in opposite quadrants being contracted or expanded, so that in passing from one quadrant to the next the colours pass into their complementaries. If either the direction of the axis of the quarter-undulation plate be changed from $45^{\circ}$ on one side to $45^{\circ}$ on the other side of the plane of vibration of the polariser; or if the crystal be changed for another of an opposite character (i.e. negative for positive, or vice versâ), the quadrants which were first contracted will be expanded, and those which were first expanded will be contracted. Hence for a given position of the quarter-undulation plate the appearance of the rings will furnish a means of determining the character of the crystal under examination.

Similar effects are produced if the quarter-undulation plate be placed between the crystal and the analyser ; that is, if the light be analysed circularly.

In the case of bi-axal crystals under the action of light polarised or analysed circularly, the black brushes are wanting, but they are replaced by lines of the same form marking where the segments of the lemniscatas pass from given colours into their complementaries.

If the light be both polarised and analysed circularly, all trace of direction will have disappeared. In uni-axal crystals the rings will take the form of perfect circles without break of any kind; and in bi-axal they will exhibit complete lemniscatas.

To pursue this matter one step farther. Suppose that, the arrangements remaining otherwise as before (viz., first, the polariser; secondly, a quarter-undulation plate with its axis at $45^{\circ}$ to the principal plane of the polariser; thirdly, a uni-axal crystal; fourthly, a quarter-undulation plate with its axis parallel or perpendicular to the first ; and, lastly, the analyser), the analyser be turned round; then in any position intermediate to $0^{\circ}$ and $90^{\circ}$ the rings will be contracted and extended in opposite quadrants until at $45^{\circ}$ they are divided by two diagonals, on each side of which the colours are complementary. Beyond $45^{\circ}$ the rings begin to coalesce, until at $90^{\circ}$ the four quadrants coincide again. During this movement the centre has changed from bright to dark. If the motion of the analyser be reversed the quadrants which before contracted now expand, and vice vers $\hat{\theta}$. Again, if the crystal be replaced by another of an opposite character, say positive for negative, the effect on the quadrants of the rings will be reversed. This method of examination, therefore, affords a test of the character of a crystal.

A similar process applies to bi-axal crystals; but in this case the diagonals interrupting the rings are replaced by a pair of rectangular hyperbolas, on either side of which the rings expand or contract, and the effect is reversed by reversing the motion of the analyser, or by replacing a positive by a negative crystal. The test experiment may then be made by turning the analyser slightly to the right or left, and observing whether the rings appear to advance to, or recede from, one another in the centre of the field. In particular if, the polariser and analyser being parallel, the first plate have its axis in a N.E. direction to a person looking through the analyser, the second plate with its axis at right angles to the former, and the crystal be so placed that the line joining the optic axes by N.S., then on turning the analyser to the right, the rings will advance towards one another if the crystal be negative, and recede if it be positive.

\section{W. SPOTTLWOODE}

